\title{
A Wide Range of FIB Applications in a Multidisciplinary Analytical Facility
}

\author{
A.D. Batchelor, D.P. Griffis and P.E. Russell \\ Materials Science and Engineering Department and Analytical Instrumentation Facility, North Carolina State \\ University, Box 7531, Raleigh, NC 27695-7531
}

The Focused Ion Beam (FIB) system is a versatile tool which can be applied to the preparation of a wide variety of materials for microscopy and microanalysis. The versatility of FIB stems from the fact that this tool can be used to both remove and add material from a substrate. In this work, a variety of non traditional (i.e. non semiconductor) applications for FIB are presented.

A Hitachi High Technologies FIB 2100 focused ion beam system was used to perform site specific modifications of a variety of samples. These modifications include both cross sectioning and materials deposition. Samples ranged from polymer nanofibers to biological cells as well as semiconductors to thin film metal and ceramic coatings. For the investigations in this work, the Ga liquid metal ion source was operated at a beam energy of $40 \mathrm{kV}$ and at beam currents ranging from $30 \mathrm{nA}$ down to $10 \mathrm{pA}$. Examples presented include analysis of collogen clad polyester nanofibers and a carbon fiber/epoxy resin composite. An additional example examines the quality of a tungsten back filled micro machined via in a gold coated $\mathrm{SiO}_{2}$ film on a silicon substrate.

To illustrate the challenges (and solutions) involved with FIB based analysis of complex non traditional samples, two examples of fiber analysis are presented. The first example presented required the cross sectioning and examination of non woven polyester nanofibers with a collogen cladding that were produced using a novel electro spinning process. The goal of the analysis was to determine the presence of, and if present, the thickness of the collogen cladding and to determine the size of the polyester core. A large area view of the sample after cross sectioning and a higher magnification view of individual fibers are presented in Figure 1. It was determined from the cross section that the selectively sputtered polyester core diameters range from $\sim 100 \mathrm{~nm}$ to $250 \mathrm{~nm}$, while the remaining intact collagen cladding thicknesses range from $\sim 100 \mathrm{~nm}$ to $200 \mathrm{~nm}$ in diameter.

A second example involves the determination of the ability of a resin filler to intimately contact embedded graphite fibers in an epoxy resin/carbon fiber composite material. This material is a candidate for aircraft applications, hence it is important to determine that the resin adheres to the fibers and that there are no voids in the resin. Figure 2 shows a large area and a higher magnification FIB induced secondary electron image of this epoxy resin/fiber composite after FIB sputtering. Figure 2 shows that the resin sputters faster than the carbon fibers thus providing the delineation required to determine that the resin/fiber contact is free of voids. Although the ends of the graphite fibers are clearly damaged by the FIB ion beam, the damage does not impact on the ability to determine the quality of the resin adhesion.

In addition to preparing cross sections of a variety of materials, the FIB can also be used to fabricate structures. For example, the FIB can be used investigate the quality of a tungsten deposition required to make electrical contact between a gold film and the Si substrate. This contact was required for a study charging effects in Secondary Ion Mass Spectrometry (SIMS) of insulators. For this study, holes and trenches as deep as 4 microns were micromachined in a gold coated $800 \mathrm{~nm} \mathrm{SiO} 2$ film on a Si substrate. These structures were then backed filled with tungsten to provide electrical contact through the oxide to the Si substrate. If these excavated features are not fully back filled with $\mathrm{W}$, poor conductivity results (Fig. 3). A technique was developed to back fill the excavated areas with tungsten in incrementally larger deposition areas to minimize voids. The lack of voids is verified by FIB cross-section (Fig. 4). 

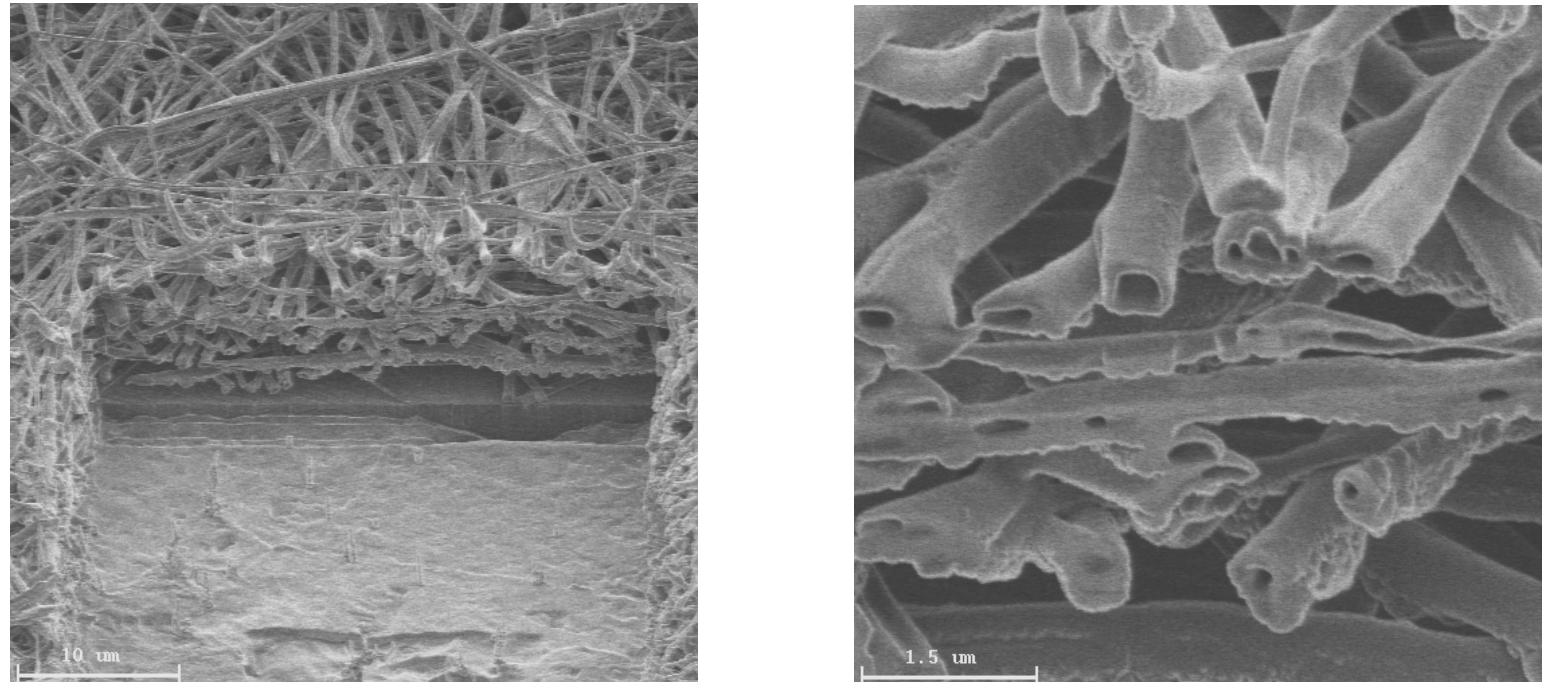

Fig. 1 FIB cross-section of electrospun non-woven collagen clad polyester nanofibers.
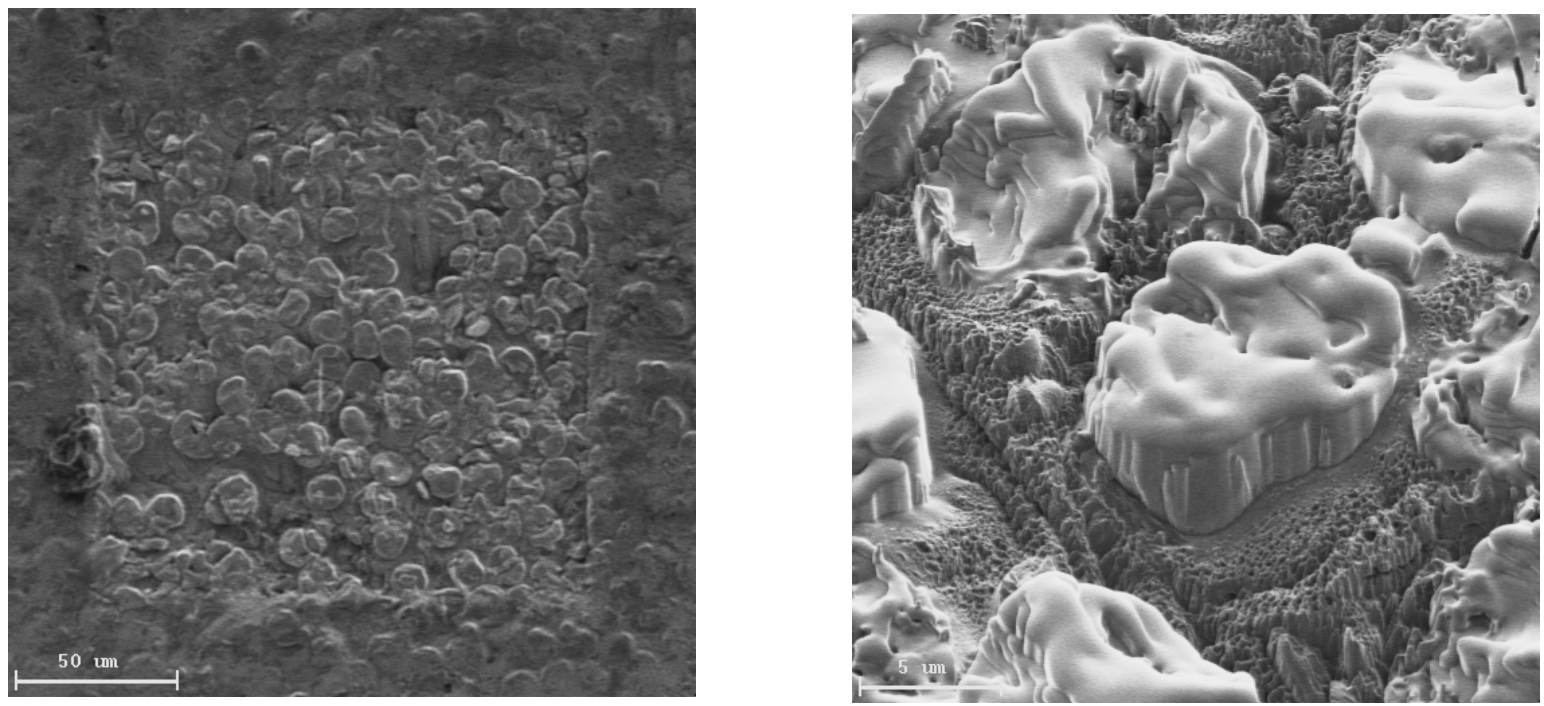

Fig. 2 FIB sputtered epoxy resin graphite fiber composite.

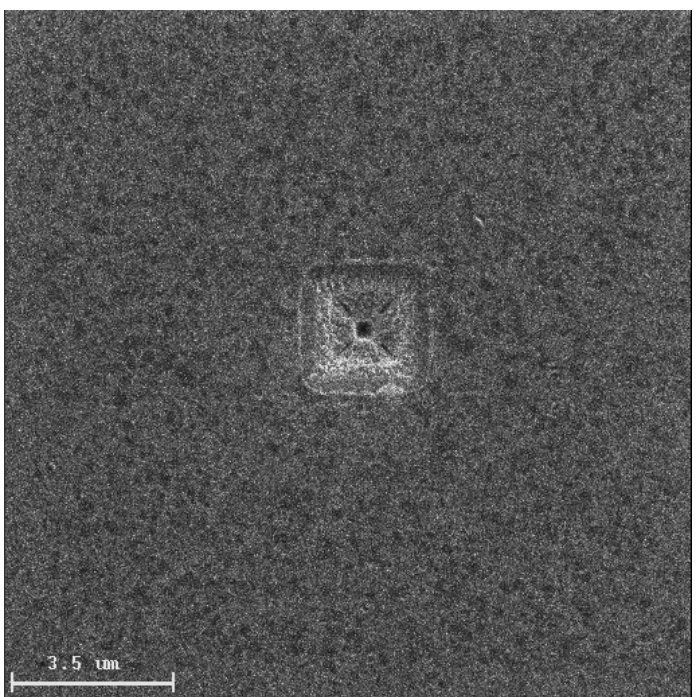

Fig. 3 FIB image of incomplete W deposition in micro-machined via.

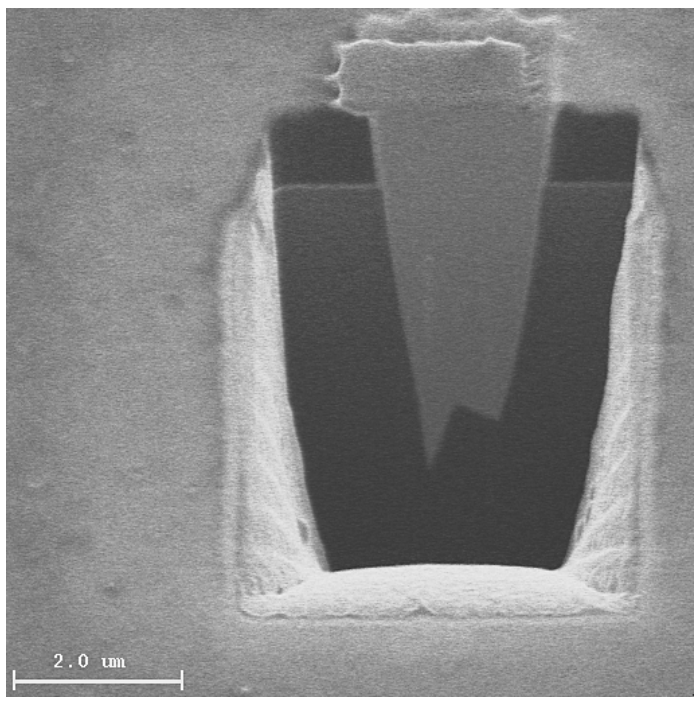

Fig. 4 FIB cross-section of W backfilled micro-machined via in gold coated $\mathrm{SiO} 2$ on Si substrate. 